\title{
On Landsberg and Berwald Spaces of Two Dimensional Finslerian Space with Special $(\alpha$, $\beta$ )-Metric
}

\author{
Pradeep Kumar1, T. S. Madhu1, B. R. Sharath² \\ ${ }^{1}$ Department of Mathematics, Acharya Institute of Technology, Soladevanahalli, Bengaluru, India \\ ${ }^{2}$ Department of Mathematics, Vemana Institute of Technology, Bengaluru, India \\ Email: pradeepget@gmail.com, madhuts2327@gmail.com, sharathbr181@gmail.com
}

How to cite this paper: Kumar, P., Madhu, T.S. and Sharath, B.R. (2019) On Landsberg and Berwald Spaces of Two Dimensional Finslerian Space with Special $(\alpha, \beta)$-Metric. Open Access Library Journal, 6: e5244. https://doi.org/10.4236/oalib.1105244

Received: February 13, 2019

Accepted: March 10, 2019

Published: March 13, 2019

Copyright $\odot 2019$ by author(s) and Open Access Library Inc.

This work is licensed under the Creative Commons Attribution International License (CC BY 4.0).

http://creativecommons.org/licenses/by/4.0/

\begin{abstract}
In the present paper, we find a condition for the two-dimensional Finsler space with a special $(\alpha, \beta)$-metric $L(\alpha, \beta)=\alpha+\frac{\alpha^{2}}{\beta}$ to be a Berwald space.
\end{abstract} Also we have proved that, if the two-dimensional Finsler space with above metric is a Landsberg space, then it is a Berwald space.

\section{Subject Areas}

Mathematical Analysis

\section{Keywords}

Finsler Space, Berwald Space, Cartan Connection, Landsberg Space, Main Scalar

\section{Introduction}

In the carton connection $C \Gamma$, if the covariant derivative $C_{h i j k}$ satisfies $C_{h i j \mid k}(x, y) y^{k}=0$ then the Finsler space is known as Landsberg space. L. Berwald introduced a class of Finslerian spaces which are known as Berwald spaces in which local coefficients of the Berwald connection depend only on position coordinates. If Landsberg space satisfy some conditions, then it is Berwald space [1]. On the other hand, in two-dimensional case, the main scalar of a general Finsler space $I(x, y)$ satisfies $I_{\mid i} y^{i}=0$ if and only if general Finslerian space is a Landsberg space [2].

The purpose of the present paper is to find a two-dimensional Landsberg space 
with a special $(\alpha, \beta)$-metric $L(\alpha, \beta)=\alpha+\frac{\alpha^{2}}{\beta}$ satisfying some conditions. First we find the condition for a Finsler space with a special $(\alpha, \beta)$-metric to be a Berwald space. Next, we determine the difference vector and the main scalar of $F^{2}$ with the aforesaid metric.

Finally, we derive the condition for a two-dimensional Finsler space $F^{2}$ with a special $(\alpha, \beta)$-metric $L(\alpha, \beta)=\alpha+\frac{\alpha^{2}}{\beta}$ to be a Landsberg space and we have shown that if $F^{2}$ with the mentioned metric is a Landsberg space, then it is a Berwald space.

\section{Preliminaries}

Let an $n$-dimensional Finsler space $F^{n}=\left(M^{n}, L(\alpha, \beta)\right)$ with $(\alpha, \beta)$-metric and the associated Riemannian space $R^{n}=\left(M^{n}, \alpha\right)$ where $\alpha^{2}=a_{i j}(x) y^{i} y^{j}, \beta=b_{i}(x) y^{i}$. We put $a^{i j}=\left(a_{i j}\right)^{-1}$ since $a_{i j}$ is invertible. In the following, we restrict our discussions to a domain of $(x, y)$ where $\beta$ does not vanish by taking Riemannian metric $\alpha$ is not supposed to be positive definite. The semi-colon denotes the covariant differentiation in the Levi-Civita connection $\left(\gamma_{j k}^{i}(x)\right)$ of $R^{n}$.

We have the following symbols

$$
\begin{gathered}
b^{i}=a^{i r} b_{r}, b^{2}=a^{r s} b_{r} b_{s}, \\
2 r_{i j}=b_{i ; j}+b_{j ; i}, 2 s_{i j}=b_{i ; j}-b_{j ; i}, \\
r_{j}^{i}=a^{i r} r_{r j}, s_{j}^{i}=a^{i r} s_{r j}, r_{i}=b_{r} r_{i}^{r}, s_{i}=b_{r} s_{i}^{r}, \\
L_{\alpha}=\partial L / \partial \alpha, L_{\beta}=\partial L / \partial \beta, L_{\alpha \alpha}=\partial^{2} L / \partial \alpha^{2}, L_{\beta \beta}=\partial^{2} L / \partial \beta^{2} .
\end{gathered}
$$

Here $B \Gamma=\left(G_{j k}^{i}, G_{j}^{i}, 0\right)$ of $F^{n}$ plays an important role. Denote by $B_{j k}^{i}$ the difference tensor of Matsumoto [3] of $G_{j k}^{i}$ from $\gamma_{j k}^{i}$ :

$$
G_{j k}^{i}(x, y)=\gamma_{j k}^{i}(x, y)+B_{j k}^{i}(x, y) .
$$

Transvecting above by $y^{i}$ and then by $y^{j}$, we have

$$
\left.\begin{array}{l}
G_{j}^{i}=\gamma_{0 j}^{i}+B_{j}^{i}, \\
2 G^{i}=\gamma_{00}^{i}+2 B^{i} .
\end{array}\right\}
$$

Then $B_{j}^{i}=\dot{\partial}_{j} B^{i}$ and $B_{j k}^{i}=\dot{\partial}_{k} B_{j}^{i}$.

On account Matsumoto [3], the components of $B_{j k}^{i}$ is determined by

$$
L_{\alpha} B_{j i}^{k} y^{i} y_{k}=\alpha L_{\beta}\left(b_{j ; i}-B_{j i}^{k} b_{k}\right) y^{j} .
$$

According to Matsumoto [3], $B^{i}(x, y)$ is called the difference vector if

$$
\beta^{2} L_{\alpha}+\alpha \gamma^{2} L_{\alpha \alpha} \neq 0 \text {, }
$$

where $\gamma^{2}=b^{2} \alpha^{2}-\beta^{2}$.

Then $B^{i}$ is written as follows

$$
B^{i}=\frac{E}{\alpha} y^{i}+\frac{\alpha L_{\beta}}{L_{\alpha}} s_{0}^{i}-\frac{\alpha L_{\alpha \alpha}}{L_{\alpha}} C\left(\frac{1}{\alpha} y^{i}-\frac{\alpha}{\beta} b^{i}\right),
$$


where

$$
E=\left(\frac{\beta L_{\beta}}{L}\right) C, C=\frac{\alpha \beta\left(r_{00} L_{\alpha}-2 \alpha s_{0} L_{\beta}\right)}{2\left(\beta^{2} L_{\alpha}+\alpha \gamma^{2} L_{\alpha \alpha}\right)} .
$$

Further, by means of M. Hashiguchi, S. Hojo and M. Matsumoto [4], we have

$$
\left.\begin{array}{l}
\alpha_{\mid i}=-\frac{L_{\beta}}{L_{\alpha}} \beta_{i i}, \\
\beta_{\mid i} y^{i}=r_{00}-2 b_{r} B^{r}, \\
b_{i i}^{2} y^{i}=2\left(r_{0}+s_{0}\right), \\
\gamma_{\mid i}^{2} y^{i}=2\left(r_{0}+s_{0}\right) \alpha^{2}-2\left(\frac{L_{\beta}}{L_{\alpha}} b^{2} \alpha+\beta\right)\left(r_{00}-2 b_{r} B^{r}\right) .
\end{array}\right\}
$$

We have the following lemmas

Lemma 2.1. [2] [5]. If $a_{i j} y^{i} y^{j}$ contains $b_{i}(x) y^{i}$ as a factor i.e. $\alpha^{2} \equiv 0(\bmod \beta)$, then the dimension $n=2$ and $b^{2}$ vanishes. In this case we have 1-form $\delta=d_{i}(x) y^{i}$ satisfying $\alpha^{2}=\beta \delta$ and $d_{i} b^{i}=2$.

Lemma 2.2. [4]. We consider the two dimension case.

1) If $b^{2} \neq 0$, then $\exists$ a sign $\epsilon= \pm 1$ and $\delta=d_{i}(x) y^{i} \quad \square \quad \alpha^{2}=\frac{\beta^{2}}{b^{2}}+\epsilon \delta^{2}$ and $d_{i} b^{i}=0$.

2) If $b^{2}=0$, then $\exists \delta=d_{i}(x) y^{i} \square \alpha^{2}=\beta \delta$ and $d_{i} b^{i}=2$.

If two functions $f(x)$ and $g(x)$ satisfies $f \alpha^{2}+g \beta^{2}=0$, then it is cleared that $f=g=0$ because $f \neq 0$ gives a contradiction $\alpha^{2}=-\frac{g}{f} \beta^{2}$.

Throughout the chapter, for brevity we shall say "homogeneous polynomial $(s)$ in $y^{i}$ of degree $r^{\prime \prime}$. Hence $\gamma_{00}^{i}$ are $h p(2)$.

\section{Berwald Space}

In this section, Let us consider an $n$-dimensional Finslerian space $F^{n}=\left(M^{n}, L(\alpha, \beta)\right)$ with the following special $(\alpha, \beta)$-metric

$$
L(\alpha, \beta)=\alpha+\frac{\alpha^{2}}{\beta} \text {. }
$$

First we shall assume $b^{2} \neq 0$.

Suppose if $b^{2}=0$, then from lemma (2.2), we have $\alpha^{2}=\beta \delta$, then $L(\alpha, \beta)=\alpha+\delta$, which is a Randers metric. So the assumption $b^{2} \neq 0$ is reasonable.

Then from the above, we have

$$
\begin{gathered}
L_{\alpha}=1+2 \frac{\alpha}{\beta}, \\
L_{\beta}=-\frac{\alpha^{2}}{\beta^{2}}, \\
L_{\alpha \alpha}=\frac{2}{\beta},
\end{gathered}
$$




$$
L_{\beta \beta}=2 \frac{\alpha^{2}}{\beta^{3}} .
$$

Substituting (3.2) into (3.3), we obtain

$$
\beta^{2} B_{j i}^{k} y^{i} y_{k}+\alpha\left\{2 \beta B_{j i}^{k} y^{i} y_{k}+\alpha^{2}\left(b_{j ; i}-B_{j i}^{k} b_{k}\right) y^{j}\right\}=0 .
$$

Assume that the Finsler space with metric (3.1) be a Berwald space, i.e., $G_{j k}^{i}=G_{j k}^{i}(x)$.

Then we have $B_{j i}^{k}=B_{j i}^{k}(x)$, so LHS of (3.3) has a form

$$
P(x, y)+\alpha Q(x, y)=0,
$$

where $P$ and $Q$ are polynomials in $y^{i}$ while $\alpha$ is irrational in $y^{i}$. Hence the Equation (3.3) shows $P=Q=0$.

By Lemma (2.1), we have

$$
B_{j i}^{k} a_{k h} y^{j} y^{h}=0,\left(b_{j ; i}-B_{j i}^{k} b_{k}\right) y^{i}=0 .
$$

The former yields $B_{j i}^{k} a_{k h}+B_{h i}^{k} a_{k j}=0$, so we have $B_{j i}^{k}=0$. Then the latter leads to $b_{j ; i}=0$ directly.

Conversely, if $b_{j ; i}=0$, by well known Okada's axioms $\left(\gamma_{j k}^{i}, \gamma_{0 j}^{i}, 0\right)$ becomes the Berwald connection of $F^{n}$. Thus $F^{n}$ is a Berwald space.

Hence we have the following result

Theorem 3.1. The Finsler space $F^{n}$ with special $(\alpha, \beta)$-metric (3.1) satisfying $b^{2} \neq 0$ is a Berwald space if and only if $b_{j ; i}=0$, then Berwald connection is essentially Riemannian $\left(\gamma_{j k}^{i}, \gamma_{0 j}^{i}, 0\right)$.

\section{Two-Dimensional Landsberg Space}

In this section, Let us consider an $n$-dimensional Finslerian space $F^{n}=\left(M^{n}, L(\alpha, \beta)\right)$ with the following special $(\alpha, \beta)$-metric

$$
L(\alpha, \beta)=\alpha+\frac{\alpha^{2}}{\beta} .
$$

By means of (2.4) and (3.2), the difference vector $B^{i}$ [6] of the Finsler space becomes

$$
2 B^{i}=\frac{A}{\beta(\beta+2 \alpha) L \Omega}\left\{B y^{i}+2 \alpha^{3} L b^{i}\right\}-\frac{2 \alpha^{3}}{\beta(\beta+2 \alpha)} s_{0}^{i},
$$

where

$$
\begin{gathered}
A=\beta(\beta+2 \alpha) r_{00}+2 \alpha^{3} s_{0}, \\
B=-3 \alpha^{2} \beta-4 \alpha^{3}, \\
\Omega=\beta^{3}+2 b^{2} \alpha^{3} .
\end{gathered}
$$

It is trivial that $\beta \neq 0, \beta+2 \alpha \neq 0$ and $\Omega \neq 0$, because $\alpha$ is irrational in $y^{i}$. From (4.2) it follows that

$$
r_{00}-2 b_{r} B^{r}=\frac{\alpha(\beta+2 \alpha) A}{L \Omega} .
$$


In two-dimensional case, the main scalar of a general Finsler space $I(x, y)$ satisfies $I_{\mid i} y^{i}=0$ if and only if general Finsler space is a Landsberg space [7]. If $F^{2}$ with (4.1), then the main scalar $I$ is obtained as follows

$$
\varepsilon I^{2}=\frac{9 \gamma^{2} M^{2}}{4 \alpha \beta L \Omega^{3}},
$$

where

$$
\begin{gathered}
M=-\alpha^{2} \beta^{3}-2 b^{2} \alpha^{4} \beta-4 b^{2} \alpha^{5}, \\
\Omega=\beta^{3}+2 b^{2} \alpha^{3} .
\end{gathered}
$$

The covariant differentiation of (4.3) leads to

$$
\begin{aligned}
4 \alpha^{2} \beta^{2} L \Omega^{4} \varepsilon I_{\mid i}^{2}= & 9 M\left(\alpha \beta \Omega M \gamma_{\mid i}^{2}+2 \alpha \beta \Omega \gamma^{2} M_{\mid i}-\beta \Omega \gamma^{2} M \alpha_{\mid i}\right. \\
& \left.-\alpha \Omega \gamma^{2} M \beta_{\mid i}-3 \alpha \beta \gamma^{2} M \Omega_{\mid i}\right) .
\end{aligned}
$$

Transvecting (4.4) by $y^{i}$, we have

$$
4 \alpha^{2} \beta^{2} L \Omega^{4} \varepsilon I_{\mid i}^{2}=9 M\left(U \gamma_{\mid i}^{2} y^{i}+Q M_{\mid i} y^{i}-R \alpha_{\mid i} y^{i}-S \beta_{\mid i} y^{i}-T \Omega_{\mid i} y^{i}\right),
$$

where

$$
\begin{array}{rl}
U= & -\alpha^{3} \beta^{7}-2 b^{2} \alpha^{5} \beta^{5}-6 b^{2} \alpha^{6} \beta^{4}-4 b^{4} \alpha^{8} \beta^{2}-8 b^{4} \alpha^{9} \beta, \\
& Q=-2 \alpha \beta^{6}+2 b^{2} \alpha^{3} \beta^{4}-4 b^{2} \alpha^{4} \beta^{3}+4 b^{4} \alpha^{6} \beta, \\
R= & \alpha^{2} \beta^{9}+b^{2} \alpha^{4} \beta^{7}+6 b^{2} \alpha^{5} \beta^{6}-2 b^{4} \alpha^{6} \beta^{5}-2 b^{4} \alpha^{7} \beta^{4} \\
& +8 b^{4} \alpha^{8} \beta^{3}-4 b^{6} \alpha^{9} \beta^{2}-8 b^{6} \alpha^{10} \beta, \\
S= & \alpha^{3} \beta^{8}+b^{2} \alpha^{5} \beta^{6}+6 b^{2} \alpha^{6} \beta^{5}-2 b^{4} \alpha^{7} \beta^{4}-2 b^{4} \alpha^{8} \beta^{3} \\
& +8 b^{4} \alpha^{9} \beta^{2}-4 b^{6} \alpha^{10} \beta-8 b^{6} \alpha^{11}, \\
T=3 & 3 \alpha^{3} \beta^{6}+3 b^{2} \alpha^{5} \beta^{4}+12 b^{2} \alpha^{6} \beta^{3}-6 b^{4} \alpha^{7} \beta^{2}-12 b^{4} \alpha^{8} \beta .
\end{array}
$$

Hence (4.5) can be put in the form

$$
4 \alpha^{2} \beta^{2} L \Omega^{4} \varepsilon I_{\mid i}^{2} y^{i}=9 M\left(U \gamma_{\mid i}^{2} y^{i}+V \alpha_{\mid i} y^{i}+W \beta_{\mid i} y^{i}+X b_{\mid i}^{2} y^{i}\right),
$$

where

$$
\begin{gathered}
V=3 \alpha^{2} \beta^{9}+11 b^{2} \alpha^{4} \beta^{7}+24 b^{2} \alpha^{5} \beta^{6}-14 b^{4} \alpha^{6} \beta^{5}-32 b^{4} \alpha^{7} \beta^{4}+8 b^{6} \alpha^{9} \beta^{2}, \\
W=-4 \alpha^{3} \beta^{8}-12 b^{2} \alpha^{5} \beta^{6}-30 b^{2} \alpha^{6} \beta^{5}+16 b^{4} \alpha^{7} \beta^{4}+34 b^{4} \alpha^{8} \beta^{3} \\
-8 b^{4} \alpha^{9} \beta^{2}-4 b^{6} \alpha^{10} \beta+8 b^{6} \alpha^{11}, \\
X=4 \alpha^{5} \beta^{7}+5 \alpha^{6} \beta^{6}-4 b^{2} \alpha^{7} \beta^{5}-3 b^{2} \alpha^{8} \beta^{4}+4 b^{2} \alpha^{9} \beta^{3}-2 b^{4} \alpha^{10} \beta^{2}-4 b^{4} \alpha^{11} \beta .
\end{gathered}
$$

Consequently, the Finslerian space $F^{2}$ with special $(\alpha, \beta)$-metric (4.1) is Landsberg space if and only if

$$
U \gamma_{\mid i}^{2} y^{i}+V \alpha_{\mid i} y^{i}+W \beta_{\mid i} y^{i}+X b_{\mid i}^{2} y^{i}=0,
$$

since $M \neq 0$.

If $M=0$, then $b^{2}=0$ which is a contradiction.

In view of (2.5), the above equation written as

$$
\begin{aligned}
& 2\left(\beta^{2}+2 \alpha \beta\right)\left(\alpha^{2} U+X\right)\left(r_{0}+s_{0}\right) \\
& +\left[V \alpha^{2}+W\left(\beta^{2}+2 \alpha \beta\right)-2\left\{\beta^{3}+2 \alpha \beta^{2}-b^{2} \alpha^{3}\right\} U\right]\left(r_{00}-2 b_{r} B^{r}\right)=0 .
\end{aligned}
$$


Substituting the values of $U, V, W, X$ and $\left(r_{00}-2 b_{r} B^{r}\right)$ in (4.6), we obtain $\alpha^{6} \beta^{3}\left[6 \beta^{10}+\left(42-12 b^{2}\right) \alpha^{2} \beta^{8}-14 \alpha^{4} \beta^{6}+\left(24 b^{2}-168 b^{4}\right) \alpha^{6} \beta^{4}\right.$ $\left.+\left(-136 b^{4}-24 b^{6}\right) \alpha^{8} \beta^{2}-192 b^{6} \alpha^{10}\right]\left(r_{0}+s_{0}\right)+\alpha^{7} \beta^{2}\left[28 \beta^{10}+\left(20-42 b^{2}\right) \alpha^{2} \beta^{8}\right.$ $\left.+\left(40 b^{2}-36 b^{4}\right) \alpha^{4} \beta^{6}-236 b^{4} \alpha^{6} \beta^{4}+\left(-120 b^{6}-32 b^{4}\right) \alpha^{8} \beta^{2}-96 b^{6} \alpha^{10}\right]\left(r_{0}+s_{0}\right)$ $+\alpha^{4} \beta^{6}\left[256 b^{4} \alpha^{8}-16 b^{6} \alpha^{8}+336 b^{4} \alpha^{6} \beta^{2}-144 b^{2} \alpha^{6} \beta^{2}-168 b^{2} \alpha^{4} \beta^{4}\right.$ $\left.+16 b^{4} \alpha^{4} \beta^{4}-12 \alpha^{2} \beta^{6}-2 \beta^{8}\right] r_{00}+\alpha^{5} \beta^{5}\left[-9 \beta^{8}-57 b^{2} \alpha^{2} \beta^{6}-4 \alpha^{2} \beta^{6}\right.$ $\left.+110 b^{4} \alpha^{4} \beta^{4}-244 b^{2} \alpha^{4} \beta^{4}+432 b^{4} \alpha^{6} \beta^{2}-4 b^{6} \alpha^{6} \beta^{2}+64 b^{4} \alpha^{8}-16 b^{6} \alpha^{8}\right] r_{00}$ $+\alpha^{8} \beta^{5}\left[-10 \beta^{6}-178 b^{2} \alpha^{2} \beta^{4}-144 b^{2} \alpha^{4} \beta^{2}+176 b^{4} \alpha^{4} \beta^{2}+224 b^{4} \alpha^{6}-8 b^{6} \alpha^{6}\right] s_{0}$ $+\alpha^{7} \beta^{4}\left[-4 \beta^{8}-16 b^{2} \alpha^{2} \beta^{6}-32 b^{4} \alpha^{4} \beta^{4}-172 b^{2} \alpha^{4} \beta^{4}+32 b^{4} \alpha^{6} \beta^{2}-16 b^{6} \alpha^{8}\right.$ $\left.+64 b^{4} \alpha^{8}\right] s_{0}=0$.

Separating (4.7) as rational and irrational terms with respect to $\left(y^{i}\right)$, we obtain

$$
\begin{aligned}
& {\left[\alpha^{6} \beta^{3} D_{1}\left(r_{0}+s_{0}\right)+\alpha^{4} \beta^{6} E_{1} r_{00}+\alpha^{8} \beta^{5} F_{1} s_{0}\right]} \\
& +\alpha\left[\alpha^{6} \beta^{2} D_{2}\left(r_{0}+s_{0}\right)+\alpha^{4} \beta^{5} E_{2} r_{00}+\alpha^{6} \beta^{4} F_{2} s_{0}\right]=0
\end{aligned}
$$

where

$$
\begin{aligned}
& D_{1}= 6 \beta^{10}+\left(42-12 b^{2}\right) \alpha^{2} \beta^{8}-14 \alpha^{4} \beta^{6}+\left(24 b^{2}-168 b^{4}\right) \alpha^{6} \beta^{4} \\
&+\left(-136 b^{4}-24 b^{6}\right) \alpha^{8} \beta^{2}-192 b^{6} \alpha^{10}, \\
& D_{2}= 28 \beta^{10}+\left(20-42 b^{2}\right) \alpha^{2} \beta^{8}+\left(40 b^{2}-36 b^{4}\right) \alpha^{4} \beta^{6}-236 b^{4} \alpha^{6} \beta^{4} \\
&+\left(-120 b^{6}-32 b^{4}\right) \alpha^{8} \beta^{2}-96 b^{6} \alpha^{10}, \\
& E_{1}= 256 b^{4} \alpha^{8}-16 b^{6} \alpha^{8}+336 b^{4} \alpha^{6} \beta^{2}-144 b^{2} \alpha^{6} \beta^{2}-168 b^{2} \alpha^{4} \beta^{4} \\
&+16 b^{4} \alpha^{4} \beta^{4}-12 \alpha^{2} \beta^{6}-2 \beta^{8}, \\
& E_{2}=-9 \beta^{8}-57 b^{2} \alpha^{2} \beta^{6}-4 \alpha^{2} \beta^{6}+110 b^{4} \alpha^{4} \beta^{4}-244 b^{2} \alpha^{4} \beta^{4} \\
&+432 b^{4} \alpha^{6} \beta^{2}-4 b^{6} \alpha^{6} \beta^{2}+64 b^{4} \alpha^{8}-16 b^{6} \alpha^{8}, \\
& F_{1}=-10 \beta^{6}-178 b^{2} \alpha^{2} \beta^{4}-144 b^{2} \alpha^{4} \beta^{2}+176 b^{4} \alpha^{4} \beta^{2}+224 b^{4} \alpha^{6}-8 b^{6} \alpha^{6}, \\
& F_{2}=-4 \beta^{8}-16 b^{2} \alpha^{2} \beta^{6}-32 b^{4} \alpha^{4} \beta^{4}-172 b^{2} \alpha^{4} \beta^{4}+32 b^{4} \alpha^{6} \beta^{2}-16 b^{6} \alpha^{8}+64 b^{4} \alpha^{8} .
\end{aligned}
$$

The Equation (4.8) yields two equations as follows

$$
\begin{aligned}
\alpha^{2} D_{1}\left(r_{0}+s_{0}\right)+\beta^{3} E_{1} r_{00}+\alpha^{4} \beta^{2} F_{1} s_{0} & =0, \\
\alpha^{2} D_{2}\left(r_{0}+s_{0}\right)+\beta^{3} E_{2} r_{00}+\alpha^{2} \beta^{2} F_{2} s_{0} & =0 .
\end{aligned}
$$

From (4.10), we obtain

$$
-9 \beta^{11} r_{00} \equiv 0\left(\bmod \alpha^{2}\right) .
$$

Then $\exists$ a function $f(x)$

$$
r_{00}=\alpha^{2} f(x)
$$

Thus, we have 


$$
r_{i j}=a_{i j} f(x) .
$$

Transvecting above by $b^{i} y^{j}$ leads to

$$
r_{0}=\beta f(x) ; r_{j}=b_{j} f(x) .
$$

Eliminating $\left(r_{0}+s_{0}\right)$ from (4.9) and (4.10), from (4.11), we have

$$
\alpha^{2} \beta^{3} f(x)\left(D_{2} E_{1}-D_{1} E_{2}\right)+\alpha^{2} \beta^{3} g(x)\left(\alpha^{2} D_{2} F_{1}-D_{1} F_{2}\right)=0 .
$$

From $\alpha^{2} \neq 0(\bmod \beta)$ it follows that $\exists$ a function $g(x) \quad \square \quad s_{0}=g(x) \beta$. Hence (4.12) is reduces to

$$
\alpha^{2} D_{2} F_{1} g(x)+\left\{f(x)\left(D_{2} E_{1}-D_{1} E_{2}\right)-g(x) D_{1} F_{2}\right\}=0 .
$$

Since only the term $-2(f(x)-12 g(x)) \beta^{18}$ of

$$
f(x)\left(D_{2} E_{1}-D_{1} E_{2}\right)-g(x) D_{1} F_{2}
$$

seemingly does not contain $\alpha^{2}$, we must have $h p(16) V_{16}$ such that $\beta^{18}=\alpha^{2} V_{16}$. Thus it is a contradiction because of $\alpha^{2} \neq 0(\bmod \beta)$, that is,

$$
f(x)\left(D_{2} E_{1}-D_{1} E_{2}\right)-g(x) D_{1} F_{2}
$$

does not contain $\alpha^{2}$ as a factor.

Thus, from (4.13) we have $g(x)=0$, which leads to $s_{0}=0$ and $s_{i}=0$. Hence

$$
f(x)\left(D_{2} E_{1}-D_{1} E_{2}\right)-g(x) D_{1} F_{2}=0,
$$

which implies $f(x)=g(x)=0$, which leads to $s_{0}=0$ and $s_{i}=0$. From (4.11), we get $r_{i j}=0$.

Summarizing up, we obtain $r_{i j}=0$ and $s_{i}=0$, that is,

$$
b_{i ; j}+b_{j ; i}=0, b^{r} b_{r ; i}=0 .
$$

Therefore $b_{i}(x)$ is the so-called killing vector field with a constant length.

According to Hashiguchi, Hojo and Matsumoto [4], the condition (4.14) is equivalent to $b_{i ; j}=0$.

Hence, we have the following result

Theorem 4.2. If a two dimensional Finsler space $F^{2}$ with a special $(\alpha, \beta)$ -metric (4.1) satisfying $b^{2} \neq 0$, is a Landsberg space then $F^{2}$ is a Berwald space.

\section{Conclusion}

In this paper, first we found a condition for a Finslerian space with special $(\alpha, \beta)$ -metric $L(\alpha, \beta)=\alpha+\frac{\alpha^{2}}{\beta}$ to be a Berwald space. Further we have proved that two-dimensional Finslerian space with a special $(\alpha, \beta)$-metric $L(\alpha, \beta)=\alpha+\frac{\alpha^{2}}{\beta}$ is a Landsberg space, then it is a Berwald space.

\section{Conflicts of Interest}

The authors declare no conflicts of interest regarding the publication of this paper. 


\section{References}

[1] Bacso, S. and Matsumoto, M. (1996) Reduction Theorems of Certain Landsberg Spaces to Berwald Spaces. Publicationes Mathematicae Debrecen, 48, 357-366.

[2] Matsumoto, M. (1986) Foundations of Finsler Geometry and Finsler Spaces. Kaiseisha Press, Japan, 520.

[3] Matsumoto, M. (1991) The Berwald Connection of Finsler with an $(\alpha, \beta)$-Metric. Tensor N. S., 50, 18-21.

[4] Hashiguchi, M., Hojo, S. and Matsumoto, M. (1996) Landsberg Spaces of Dimension Two with $(\alpha, \beta)$-Metric. Tensor N. S., 57, 145-153.

[5] Bacso, S. and Matsumoto, M. (1994) Projective Change Finsler Space with $(\alpha, \beta)$ -Metrics. IBIBD, 55, 252-257.

[6] Shibata, C., Shimada, H., Azuma, M. and Yasuda, H. (1977) On Finsler Spaces with Randers Metric. Tensor N. S., 31, 252-257.

[7] Kitayama, M., Azuma, M. and Matsumoto, M. (1995) On Finsler Spaces with $(\alpha, \beta)$-Metric, Regularity, Geodesics and Main Scalars. Journal of Hokkaido University of Education Section II A, 46, 1-10. 